\title{
ON THE ULAM-HYERS STABILITIES OF THE SOLUTIONS OF Y-HILFER FRACTIONAL DIFFERENTIAL EQUATION WITH ABSTRACT VOLTERRA OPERATOR
}

\author{
1 J. VANTERLER DA C. SOUSA, ${ }^{2}$ KISHOR D. KUCCHE, \\ AND ${ }^{3}$ E. CAPELAS DE OLIVEIRA
}

\begin{abstract}
In this paper, we consider the new class of the fractional differential equation involving the abstract Volterra operator in the Banach space and investigate existence, uniqueness and stabilities of Ulam-Hyers on the compact interval $\Delta=[a, b]$ and on the infinite interval $I=[a, \infty)$. Our analysis is based on the application of the Banach fixed point theorem and the Gronwall inequality involving generalized $\Psi$-fractional integral. At last, we performed out an application to elucidate the outcomes got.
\end{abstract}

\section{INTRODUCTION}

The study of abstract Volterra equations has long been investigated by a number of researchers such as Kai-Jaung Pei [1] and Corduneanu 2, 3, 4. In 2004, Vath [5] introduced more general versions of the linear and nonlinear abstract Volterra operator and investigated the existence of local solutions and solution extensions. In 2000, Bedivan and O'Regan [6] investigated the topological structure of the set of solutions for abstract Volterra equations in the Banach and Fréchet spaces. In 2010, Serban, Rus and Petrusel [7] investigated the existence, data dependence and comparison results for the solution, using the technique of the operator of Picard. With the progression of time, studies related to the Volterra abstract operator gained interest from a number of consultants, and with this, numerous applications have emerged, such as: control theory, continuous mechanics, nuclear reactor dynamics, linear viscoelastic, among others [8, 9, 10.

On the other hand, the stability investigation of differential and integral equations are important in applications. Hyers, Isac and Rassias [1] investigated the stability of functional equations in several variables. Jung [12] carried out a work involving fixed point for stability of integral equations of Volterra. Two interesting and important works were investigated by Rassias [13] and Rus [14, in which they dealt with the stability of a linear mappings in the Banach space and the Ulam stability of ordinary differential equations. It is also important to highlight the work carried out by Otrocol [15] on Ulam stability of nonlinear differential equations with the abstract Volterra operator in the Banach spaces.

In 2012, Wei, Li and Li [16] investigated new Ulam-Hyers stabilities of Volterra integral equations by means of the fixed-point theorem. Kostic [17] has done a work on abstract Volterra integro-differential equations by means of fractional derivative

Date: January 1, 2001 and, in revised form, June 22, 2001.

2000 Mathematics Subject Classification. Primary 26A33; Secondary 34L05, 34K20, 47H10.

Key words and phrases. $\Psi$-Hilfer fractional derivative, existence, uniqueness, Ulam-Hyers stability, abstract Volterra operator, Gronwall inequality. 
and integral. Other important and interesting works on existence, uniqueness and Ulam-Hyers stabilities, we suggest the references [18, 19].

The fractional calculus over the time has been gaining increasing prominence in the scientific community $20,21,22,23$. With new definitions of fractional derivatives and integrals [24, 25, there has arisen some applications in a few territories of learning [26, 27, 28, 29, 30, 31. There has gained prominence interest in the investigation of existence and Ulam-Hyers stabilities of differential equations and fractional integrals, be they of the impulsive type, functional or of Volterra 18, 32, 33, 34, 35. Recently, Sousa et al. 36, 37, 38, 39, 40, 41, 42, investigated the existence, uniqueness and stabilities of Ulam-Hyers of fractional differential equations.

In this sense, by means of the $\Psi$-Hilfer fractional derivative, we introduced a class of nonlinear fractional differential equation with the abstract Volterra operator, in order to investigate a new class of Ulam-Hyers stabilities, contributing with new results and consequently for the growth of the area.

Consider the fractional differential equation with abstract Volterra operator in a Banach space given by

$$
\left.{ }^{\mathbf{H}} \mathbf{D}_{a^{+}}^{\mu, \eta, \Psi} x(t)=f(t, x(t), \widetilde{\mathfrak{W}}(x)(t)]\right), \quad t \in \Delta \subset \mathbb{R}
$$

where ${ }^{\mathbf{H}} \mathbf{D}_{a^{+}}^{\mu, \eta, \Psi}(\cdot)$ is the $\Psi$-Hilfer fractional derivative of order $0<\mu \leq 1$ and type $0 \leq \eta \leq 1, \Delta=[a, b]$ or $I=[a, \infty), \widetilde{\mathfrak{W}} \in C\left(\left(C_{1-\xi ; \Psi}[a, b], \mathbb{R}\right),\left(C_{1-\xi ; \Psi}[a, b], \mathbb{R}\right)\right)$, $f \in\left(I \times \mathbb{R}^{2}, \mathbb{R}\right)$, where $(\mathbb{R},|\cdot|)$ is a Banach space.

Proposing new results on existence, uniqueness, especially Ulam-Hyers stabilities, is indeed very important and contributes significantly to mathematics, especially for fractional calculus. The fundamental inspiration for the elaboration of the present work for Eq. (1.1) involving abstract Volterra operator originated in order to investigate and propose existence, uniqueness and stability results of Ulam-Hyers and Ulam-Hyers-Rassias.

This paper is organized as follows. In section 2 we present the space of the weighted functions and their respective norm. In addition, we present the concepts of $\Psi$-Riemann-Liouville fractional integral, $\Psi$-Hilfer fractional derivative, the fundamental results in this regard and the Gronwall theorems. The stability concepts of Ulam-Hyers, Ulam-Hyers-Rassias and some important observations conclude the section. In section 3, we investigated the existence, uniqueness and Ulam-Hyers stability of the fractional differential equation Eq.(1.1) on the compact interval $\Delta=[a, b]$. Section 4 is intended to investigate the uniqueness and Ulam-HyersRassias stability on the infinite interval $I=[a, \infty)$. In section 5 , as an application of the results we obtained, we will discuss existence and Ulam-Hyers stability of solution for fractional Cauchy problem involving the Hadamard derivative. The concluding remarks close the paper.

\section{Preliminaries}

In this section, we introduce the space of weighted functions and their respective norm. We introduce the concepts of $\Psi$-Riemann-Liouville fractional integral, $\Psi$ Hilfer fractional derivative and the fixed-point theorem of Banach. In addition, we present the generalized Gronwall theorems to investigate the stabilities of UlamHyers and Ulam-Hyers-Rassias. Some important remarks close the section. 
ON THE ULAM-HYERS STABILITIES OF THE SOLUTIONS OF $\Psi$-HILFER FRACTIONAL DIFFERENTIAL EQUATION WITH

Let $\Delta=[a, b]$ or $I=[a, \infty)$ and $C(\Delta, \mathbb{R})$ the space of continuous functions with norm [24, 36]

$$
\|x\|=\sup _{t \in \Delta}|x(t)| .
$$

The weighted space $C_{1-\xi, \Psi}(I, \mathbb{R})$ of functions $x$ is defined by 24,36 ,

$$
C_{1-\xi, \Psi}(\Delta, \mathbb{R})=\left\{x \in C\left(I_{2}, \mathbb{R}\right),(\Psi(t)-\Psi(a))^{1-\xi} x(t) \in C(\Delta, \mathbb{R})\right\},
$$

where $0 \leq \xi \leq 1$ with norm

$$
\|x\|_{C_{1-\xi, \Psi}}=\sup _{t \in \Delta}\left|(\Psi(t)-\Psi(a))^{1-\xi} x(t)\right| .
$$

Obviously, the space $C_{1-\xi ; \Psi}(\Delta, \mathbb{R})$ is a Banach space. The weighted space $C_{1-\xi ; \Psi}^{n}(\Delta, \mathbb{R})$ of functions $x$ is defined by

$$
C_{1-\xi, \Psi}^{n}(\Delta, \mathbb{R})=\left\{x:(a, b] \rightarrow \mathbb{R} ; x(t) \in C^{n-1}\left(\Delta, \mathbb{R} ; x^{(n)}(t) \in C_{1-\xi, \Psi}(\Delta, \mathbb{R})\right\},\right.
$$

where $0 \leq \xi \leq 1$, with norm

$$
\|x\|_{C_{1-\xi, \Psi}^{n}(\Delta, \mathbb{R})}=\sum_{k=0}^{n-1}\left\|x^{(k)}\right\|_{C(\Delta, \mathbb{R})}+\left\|x^{(n)}\right\|_{C_{1-\xi, \Psi}(\Delta, \mathbb{R})} .
$$

Let $I_{2}=(a, b)(-\infty \leq a<b \leq \infty)$ be a finite or infinite interval of the real line $\mathbb{R}$ and let $\mu>0$. Also, let $\Psi(t)$ be an increasing and positive monotone function on $I_{3}=(a, b]$, having a continuous derivative $\Psi^{\prime}(t)$ on $I_{1}$. The left-sided fractional integral of a function $f$ with respect to function $\Psi$ on $[a, b]$ is defined by 24]

$$
\mathbf{I}_{a^{+}}^{\mu, \Psi} x(t)=\frac{1}{\Gamma(\mu)} \int_{a}^{t} \mathbf{Q}_{\Psi}^{\mu}(t, s) x(s) \mathrm{d} s,
$$

where $\mathbf{Q}_{\Psi}^{\mu}(t, s):=\Psi^{\prime}(s)(\Psi(t)-\Psi(s))^{\mu-1}$. The right-sided fractional integral is defined in an analogous form.

On the other hand, let $n-1<\mu \leq n$ with $n \in \mathbb{N}, \Delta=[a, b]$ an interval such that $(-\infty \leq a<b \leq \infty)$ and let $f, \Psi \in C^{n}(\Delta, \mathbb{R})$ be two functions such that $\Psi$ is increasing and $\Psi^{\prime}(t) \neq 0$, for all $t \in \Delta$. The left-sided $\Psi$-Hilfer fractional derivative ${ }^{\mathbf{H}} \mathbf{D}_{a^{+}}^{\mu, \eta, \Psi}(\cdot)$ of a function $f$, of order $\mu(0<\mu \leq 1)$ and type $\eta(0 \leq \eta \leq 1)$, is defined by 24]

$$
{ }^{\mathbf{H}} \mathbf{D}_{a^{+}}^{\mu, \eta, \Psi} x(t)=\mathbf{I}_{a^{+}}^{\eta(n-\mu), \Psi}\left(\frac{1}{\Psi^{\prime}(t)} \frac{\mathrm{d}}{\mathrm{d} t}\right)^{n} \mathbf{I}_{a+}^{(1-\eta)(n-\mu), \Psi} x(t) .
$$

The right-sided $\Psi$-Hilfer fractional derivative is defined in an analogous form.

Theorem 1. 24] If $x \in C_{1-\xi, \Psi}^{1}(\Delta, \mathbb{R}), 0<\mu \leq 1$ and $0 \leq \eta \leq 1$, then

$$
\mathbf{I}_{a^{+}}^{\mu, \Psi} \mathbf{H} \mathbf{D}_{a^{+}}^{\mu, \eta, \Psi} x(t)=x(t)-\mathbf{M}_{\xi}^{\Psi}(t, a) \mathbf{I}_{a^{+}}^{(1-\eta)(1-\mu), \Psi} x(a),
$$

where $\mathbf{M}_{\xi}^{\Psi}(t, a):=\frac{(\Psi(t)-\Psi(a))^{\xi-1}}{\Gamma(\xi)}$.

Theorem 2. 24 Let $x \in C_{1-\xi, \Psi}^{1}(\Delta, \mathbb{R}), \mu>0$ and $0 \leq \eta \leq 1$, then we have

$$
\mathbf{D}_{a^{+}}^{\mu, \eta, \Psi} \mathbf{I}_{a^{+}}^{(1-\eta)(1-\mu), \Psi} x(t)=x(t) .
$$


$4^{1}$ J. VANTERLER DA C. SOUSA, ${ }^{2}$ KISHOR D. KUCCHE, AND ${ }^{3}$ E. CAPELAS DE OLIVEIRA

Theorem 3. 39 Let $(X, d)$ be a generalized complete metric space. Assume that $\Omega: X \rightarrow X$ is a strictly contractive operator with the Lipschitz constant $L<1$. If there exists a nonnegative integer $k$ such that $d\left(\Omega^{k+1}, \Omega^{k}\right)<\infty$ for some $x \in X$, then the following are true:

(1) The sequence $\left\{\Omega^{k} x\right\}$ converges to a point $x^{*}$ of $\Omega$;

(2) $x^{*}$ is the unique fixed point of $\Omega$ in $\Omega^{*}=\left\{y \in X / d\left(\Omega^{k} x, y\right)<\infty\right\}$;

(3) If $y \in X^{*}$, then $d\left(y, x^{*}\right) \leq \frac{1}{1-L} d(\Omega y, y)$.

Theorem 4. 43 (GRonwall theOREM). Let $u, v$ be two integrable functions and $g$ a continuous function, it domain $[a, b]$. Let $\Psi \in C^{1}(\Delta, \mathbb{R})$ an increasing function such that $\Psi^{\prime}(t) \neq 0, \forall t \in \Delta$. Assume that:

(1) $u$ and $v$ are nonnegative;

(2) $y$ is nonnegative and nondecreasing.

If

$$
u(t) \leq v(t)+g(t) \int_{a}^{b} \mathbf{Q}_{\Psi}^{\mu}(t, s) u(s) \mathrm{d} s
$$

then

$$
u(t) \leq v(t)+\int_{a}^{b} \sum_{k=1}^{\infty} \frac{[g(t) \xi(\mu)]^{k}}{\Gamma(\mu k)} \mathbf{Q}_{\Psi}^{k \mu}(t, s) v(s) \mathrm{d} s,
$$

$\forall t \in \Delta$ and $\mathbf{Q}_{\Psi}^{k \mu}(t, s):=\Psi^{\prime}(s)(\Psi(t)-\Psi(s))^{k \mu-1}$.

Lemma 1. 43. (Gronwall Lemma) Under the hypotheses of Theorem 4 let $v$ be a non-decreasing function on $\Delta$. Then, we have

$$
u(t) \leq v(t) \mathcal{E}_{\mu}\left(g(t) \Gamma(\mu)\left[(\Psi(t)-\Psi(a))^{\mu}\right]\right)
$$

$t \in \Delta$, where $\mathcal{E}_{\mu}(\cdot)$ is the Mittag-Leffler function with one parameter.

Let $(\mathbb{R},|\cdot|)$ be a Banach space and $\widetilde{\mathfrak{W}}:\left(C_{1-\xi ; \Psi} \Delta, \mathbb{R}\right) \rightarrow\left(C_{1-\xi ; \Psi} \Delta, \mathbb{R}\right)$ an abstract Volterra operator.

For $f \in C_{1-\xi ; \Psi}\left(\Delta \times \mathbb{R}^{2}, \mathbb{R}\right), \varepsilon>0$ and $\varphi \in C_{1-\xi, \Psi}\left(\Delta, \mathbb{R}_{+}\right)$we consider the following fractional Cauchy problem

$$
\begin{aligned}
{ }^{\mathbf{H}} \mathbf{D}_{a^{+}}^{\mu, \eta, \Psi} x(t) & =f(t, x(t), \widetilde{\mathfrak{W}}(x)(t)) \\
\mathbf{I}_{a^{+}}^{1-\xi, \Psi} x(a) & =\delta, \delta \in \mathbb{R}
\end{aligned}
$$

and the inequalities given below

$$
\begin{gathered}
\mid{ }^{\mathbf{H}} \mathbf{D}_{a^{+}}^{\mu, \eta, \Psi} y(t)-f(t, y(t), \widetilde{\mathfrak{W}}(y)(t) \mid \leq \varepsilon, \quad t \in \Delta, \\
\mid{ }^{\mathbf{H}} \mathbf{D}_{a^{+}}^{\mu, \eta, \Psi} y(t)-f(t, y(t), \widetilde{\mathfrak{W}} y(t) \mid \leq \varphi(t), \quad t \in \Delta .
\end{gathered}
$$

To deal with different kinds of Ulam types stabilities of Eq.(2.7) we adopt the definitions of [15.

Definition 2.1. The Eq.(2.7) is Ulam-Hyers stable if there exist a real number $c>0$ such that for each $\varepsilon>0$ and for each solution $y \in C_{1-\xi, \Psi}^{1}(\Delta, \mathbb{R})$ of inequality (2.9) there exist a solution $x \in C_{1-\xi, \Psi}^{1}(\Delta, \mathbb{R})$ of Eq.( 2.7) with

$$
\|y-x\|_{C_{1-\xi ; \Psi}(\Delta, \mathbb{R})} \leq c \varepsilon \text {. }
$$


ON THE ULAM-HYERS STABILITIES OF THE SOLUTIONS OF $\Psi$-HILFER FRACTIONAL DIFFERENTIAL EQUATION WITH

Definition 2.2. The Eq.(2.7) is generalized Ulam-Hyers-Rassias stable with respect to $\varphi$, if there exist $c_{\varphi}>0$ such that for each solution $y \in C_{1-\xi, \Psi}^{1}(\Delta, \mathbb{R})$ of inequality (2.10) with

$$
(\Psi(t)-\Psi(a))^{1-\xi}|y(t)-x(t)| \leq c_{\varphi} \varphi(t), t \in I .
$$

Remark 2.3. A function $y \in C_{1-\xi, \Psi}^{1}(\Delta, \mathbb{R})$ satisfies inequality (2.9) if and only if there exists a function $g \in C_{1-\xi, \Psi}(\Delta, \mathbb{R})$ such that

(1) $|g(t)| \leq \varepsilon, \quad t \in \Delta$

(2) ${ }^{\mathbf{H}} \mathbf{D}_{a^{+}}^{\mu, \eta}, \Psi(t)=f(t, y(t), \widetilde{\mathfrak{W}}(y)(t))+g(t), \quad t \in \Delta$.

Remark 2.4. A function $y \in C_{1-\xi, \Psi}^{1}(\Delta, \mathbb{R})$ satisfies inequality (2.10) if and only if there exists a function $\widetilde{g} \in C(\Delta, \mathbb{R})$ (which depends on $y$ ) such that

(1) $|\widetilde{g}(t)| \leq \varphi(t), \quad t \in \Delta$;

(2) ${ }^{\mathbf{H}} \mathbf{D}_{a^{+}}^{\mu, \eta}, \Psi(t)=f(t, y(t), \widetilde{\mathfrak{W}}(y)(t))+\widetilde{g}(t), \quad t \in \Delta$.

Remark 2.5. If $y \in C_{1-\xi, \Psi}^{1}(\Delta, \mathbb{R})$ satisfies inequality (2.9), then $y$ is a solution of the following integral equation

$$
\begin{aligned}
& \mid y(t)-y(a) \mathbf{M}_{\xi}^{\Psi}(t, a) \Gamma(\xi)-\frac{1}{\Gamma(\mu)} \int_{a}^{t} \mathbf{Q}_{\Psi}^{\mu}(t, s) f(s, y(s), \widetilde{\mathfrak{W}}(y)(s) \mathrm{d} s \mid \\
\leq & \frac{(\Psi(t)-\Psi(a))^{\mu}}{\Gamma(\mu+1)} \varepsilon, \quad t \in \Delta .
\end{aligned}
$$

Remark 2.6. If $y \in C_{1-\xi, \Psi}^{1}(\Delta, \mathbb{R})$ satisfies inequality (2.10), then $y$ is a solution of the following integral equation

$$
\begin{aligned}
& \mid y(t)-y(a) \mathbf{M}_{\xi}^{\Psi}(t, a)-\frac{1}{\Gamma(\mu)} \int_{a}^{t} \mathbf{Q}_{\Psi}^{\mu}(t, s) f(s, y(s), \widetilde{\mathfrak{W}}(y)(s) \mathrm{d} s \mid \\
\leq & \frac{1}{\Gamma(\mu)} \int_{a}^{b} \mathbf{Q}_{\Psi}^{\mu}(t, s) \varphi(s) \mathrm{d} s, \quad t \in \Delta .
\end{aligned}
$$

\section{UlAM-HyERS STABILITY}

In this section, our main results investigate the existence, uniqueness and stability of Ulam-Hyers of the Cauchy fractional problem (2.7)-(2.8) on the compact interval $\Delta=[a, b]$.

Theorem 5. Consider the following;

(a) $f \in C_{1-\xi, \Psi}\left(\Delta \times \mathbb{R}^{2}, \mathbb{R}\right), \widetilde{\mathfrak{W}} \in C\left(\left(C_{1-\xi, \Psi} \Delta, \mathbb{R}\right),\left(C_{1-\xi, \Psi} \Delta, \mathbb{R}\right)\right)$;

(b) There exist $L_{f}>0$ such that

$$
\left|f\left(t, u_{1}, u_{2}\right)-f\left(t, v_{1}, v_{2}\right)\right| \leq L_{f} \sum_{k=1}^{2}\left|u_{i}-v_{i}\right|, t \in[a, b],
$$

with $u_{i}, v_{i} \in \mathbb{R}, i=1,2$;

(c) There exists $L_{\widetilde{\mathfrak{W}}}>0$ such that

$$
|\widetilde{\mathfrak{W}}(x)(t)-\widetilde{\mathfrak{W}}(y)(t)| \leq L_{\widetilde{\mathfrak{W}}}|x(t)-y(t)|, \quad x, y \in C_{1-\xi, \Psi}[a, b], t \in \Delta ;
$$

(d) The inequality $L_{f}\left\{1+L_{\widetilde{\mathfrak{W}}}\right\} \frac{\Gamma(\xi)(\Psi(b)-\Psi(a))^{\mu}}{\Gamma(\xi+\mu)}<1$ is true.

Then, we have 
$6^{1}$ J. VANTERLER DA C. SOUSA, ${ }^{2}$ KISHOR D. KUCCHE, AND ${ }^{3}$ E. CAPELAS DE OLIVEIRA

(i) The fractional problem Eq.(2.7) and Eq.(2.8) has a unique solution in $C_{1-\xi, \Psi}(\Delta, \mathbb{R})$;

(ii) The solution of Eq.(2.7) is Ulam-Hyers stable.

Proof. (i). Under condition (a), Eq.(2.7) and Eq.(2.8) are equivalent to the integral equation

$$
x(t)=\mathbf{M}_{\xi}^{\Psi}(t, a) \delta+\frac{1}{\Gamma(\mu)} \int_{a}^{t} \mathbf{Q}_{\Psi}^{\mu}(t, s) f(s, x(s), \widetilde{\mathfrak{W}}(x)(s)) \mathrm{d} s .
$$

In fact, applying the integral operator $\mathbf{I}_{a_{+}}^{\mu, \Psi}(\cdot)$ on both sides of Eq.(2.7), using the relation $\mathbf{I}_{a_{+}}^{1-\xi, \Psi} x(a)=\delta$ and Theorem 1, we have

$$
x(t)-\mathbf{M}_{\xi}^{\Psi}(t, a) \mathbf{I}_{a_{+}}^{(1-\eta)(1-\mu), \Psi} x(a)=\mathbf{I}_{a+}^{\mu, \Psi} f(t, x(t), \widetilde{\mathfrak{W}}(y)(t))
$$

which implies that

$$
x(t)=\mathbf{M}_{\xi}^{\Psi}(t, a) \delta+\mathbf{I}_{a_{+}}^{\mu, \Psi} f(t, x(t), \widetilde{\mathfrak{W}}(y)(t)) .
$$

On the other hand, applying the $\Psi$-Hilfer fractional derivative ${ }^{\mathbf{H}} \mathbf{D}_{a_{+}}^{\mu, \eta, \Psi}(\cdot)$ on both sides of Eq.(3.2) and using Theorem 2, we have

$$
\begin{aligned}
{ }^{\mathbf{H}} \mathbf{D}_{a_{+}}^{\mu, \eta, \Psi} x(t) & ={ }^{\mathbf{H}} \mathbf{D}_{a_{+}}^{\mu, \eta, \Psi}\left[\mathbf{M}_{\xi}^{\Psi}(t, a) \delta+\mathbf{I}_{a+}^{\mu, \Psi} f(t, x(t), \widetilde{\mathfrak{W}}(y)(t))\right] \\
& =f(t, x(t), \widetilde{\mathfrak{W}}(y)(t))
\end{aligned}
$$

where

with $0<\xi<1$.

$$
{ }^{\mathbf{H}} \mathbf{D}_{a_{+}}^{\mu, \eta, \Psi}\left[\mathbf{M}_{\xi}^{\Psi}(t, a) \delta\right]=0
$$

Now, consider $X=C_{1-\xi, \Psi}(\Delta, \mathbb{R})$ and the operator $\mathscr{B}_{f}: X \rightarrow X$ given by

$$
\mathscr{B}_{f} x(t)=\mathbf{M}_{\xi}^{\Psi}(t, a) \delta+\frac{1}{\Gamma(\mu)} \int_{a}^{t} \mathbf{Q}_{\Psi}^{\mu}(t, s) f(s, x(s), \widetilde{\mathfrak{W}}(y)(s)) \mathrm{d} s .
$$

The main purpose here is to prove that $\mathscr{B}_{f}$ is a contraction on $X$ with respect to the norm $\|\cdot\|_{C_{1-\xi, \Psi}}$.

For any $x, y \in C_{1-\xi, \Psi}(\Delta, \mathbb{R})$ and $t \in \Delta=[a, b]$, we have

$$
\begin{aligned}
& \left|\mathscr{B}_{f}(x(t))-\mathscr{B}_{f}(y(t))\right| \\
\leq & \frac{1}{\Gamma(\mu)} \int_{a}^{t} \mathbf{Q}_{\Psi}^{\mu}(t, s) \mid f(s, x(s), \widetilde{\mathfrak{W}}(x)(s)-f(s, y(s), \widetilde{\mathfrak{W}}(y)(s) \mid \mathrm{d} s \\
\leq & \frac{1}{\Gamma(\mu)} \int_{a}^{t} \mathbf{Q}_{\Psi}^{\mu}(t, s) L_{f}\{|x(s)-y(s)|+|\widetilde{\mathfrak{W}}(x)(s)-\widetilde{\mathfrak{W}}(y)(s)|\} \mathrm{d} s \\
\leq & L_{f}\left\{1+L_{\widetilde{\mathfrak{W}}}\right\} \frac{1}{\Gamma(\mu)} \int_{a}^{t} \mathbf{Q}_{\Psi}^{\mu}(t, s)|x(s)-y(s)| \mathrm{d} s \\
\leq & L_{f}\left\{1+L_{\widetilde{\mathfrak{W}}}\right\}\|x-y\|_{C_{1-\xi ; \Psi}(\Delta, \mathbb{R})} \frac{1}{\Gamma(\mu)} \int_{a}^{t} \mathbf{Q}_{\Psi}^{\mu}(t, s)(\Psi(s)-\Psi(a))^{\xi-1} \mathrm{~d} s \\
= & L_{f}\left\{1+L_{\widetilde{\mathfrak{W}}}\right\}\|x-y\|_{C_{1-\xi ; \Psi}(\Delta, \mathbb{R})} \mathbf{I}_{a+}^{\mu ; \Psi}(\Psi(t)-\Psi(a))^{\xi-1} \\
= & L_{f}\left\{1+L_{\widetilde{\mathfrak{W}}}\right\}\|x-y\|_{C_{1-\xi ; \Psi}(\Delta, \mathbb{R})} \frac{\Gamma(\xi)(\Psi(t)-\Psi(a))^{\mu+\xi-1}}{\Gamma(\mu+\xi)}
\end{aligned}
$$


ON THE ULAM-HYERS STABILITIES OF THE SOLUTIONS OF $\Psi$-HILFER FRACTIONAL DIFFERENTIAL EQUATION WITH

Therefore, we get

$$
\begin{aligned}
& \left\|\mathscr{B}_{f} x-\mathscr{B}_{f} y\right\|_{C_{1-\xi} ; \Psi} \\
= & \sup _{t \in I}\left|(\Psi(t)-\Psi(a))^{1-\xi} \mathscr{B}_{f} x(t)-\mathscr{B}_{f} y(t)\right| \\
\leq & L_{f}\left\{1+L_{\widetilde{\mathfrak{W}}}\right\}\|x-y\|_{C_{1-\xi ; \Psi}(\Delta, \mathbb{R})} \frac{\Gamma(\xi)}{\Gamma(\mu+\xi)} \sup _{t \in I}(\Psi(t)-\Psi(a))^{\mu} \\
\leq & L_{f}\left\{1+L_{\widetilde{\mathfrak{W}}}\right\} \frac{\Gamma(\xi)}{\Gamma(\mu+\xi)}(\Psi(b)-\Psi(a))^{\mu}\|x-y\|_{C_{1-\xi ; \Psi}(\Delta, \mathbb{R})} .
\end{aligned}
$$

Condition (d) ensures that $\mathscr{B}_{f}$ is a contraction with the norm $\|\cdot\|_{C_{1-\xi, \Psi}(\Delta, \mathbb{R})}$ on $C_{1-\xi, \Psi}(\Delta, \mathbb{R})$. Thus, by means of Banach fixed point (Theorem 3) the problem Eq.(2.7) and Eq.(2.8) has a unique solution in $C_{1-\xi, \Psi}(\Delta, \mathbb{R})$. This concludes the first part of the proof.

(ii) Now, we consider the $y \in C_{1-\xi, \Psi}^{1}(\Delta, \mathbb{R})$ satisfying the fractional inequality Eq.(2.9) and we denote by $x \in C_{1-\xi, \Psi}^{1}(\Delta, \mathbb{R})$ the unique solution to the fractional Cauchy problem

$$
\left\{\begin{aligned}
{ }^{\mathbf{H}} \mathbf{D}_{a^{+}}^{\mu, \eta ;} x(t) & =f(t, x(t), \widetilde{\mathfrak{W}}(x)(t)), t \in \Delta \\
\mathbf{I}_{a+}^{1-\xi ; \Psi} x(a) & =y(a) .
\end{aligned}\right.
$$

Using the condition (a), we obtain

$$
x(t)=\mathbf{M}_{\xi}^{\Psi}(t, a) y(a)+\frac{1}{\Gamma(\mu)} \int_{a}^{t} \mathbf{Q}_{\Psi}^{\mu}(t, s) f(x, x(s), \widetilde{\mathfrak{W}}(x)(s)) \mathrm{d} s,
$$

for $t \in \Delta$. Since $y \in C_{1-\xi, \Psi}^{1}(\Delta, \mathbb{R})$ satisfies inequality Eq.(2.9), by means of the Remark 2.5, we have

$$
\begin{aligned}
& \left|y(t)-y(a) \mathbf{M}_{\xi}^{\Psi}(t, a)-\frac{1}{\Gamma(\mu)} \int_{a}^{t} \mathbf{Q}_{\Psi}^{\mu}(t, s) f(s, y(s), \widetilde{\mathfrak{W}}(y)(s)) \mathrm{d} s\right| \\
\leq & \frac{(\Psi(t)-\Psi(a))^{\mu}}{\Gamma(\mu+1)} \varepsilon .
\end{aligned}
$$

Therefore for $t \in[a, b]$, we can write

$$
\begin{aligned}
& |y(t)-x(t)| \\
= & \left|y(t)-\mathbf{M}_{\xi}^{\Psi}(t, a) y(a)-\frac{1}{\Gamma(\mu)} \int_{a}^{t} \mathbf{Q}_{\Psi}^{\mu}(t, s) f(s, x(s), \widetilde{\mathfrak{W}}(x)(s)) \mathrm{d} s\right| \\
\leq & \left|y(t)-\mathbf{M}_{\xi}^{\Psi}(t, a) y(a)-\frac{1}{\Gamma(\mu)} \int_{a}^{t} \mathbf{Q}_{\Psi}^{\mu}(t, s) f(s, y(s), \widetilde{\mathfrak{W}}(y)(s)) \mathrm{d} s\right|+ \\
& +\frac{1}{\Gamma(\mu)} \int_{a}^{t} \mathbf{Q}_{\Psi}^{\mu}(t, s)|f(s, y(s), \widetilde{\mathfrak{W}}(y)(s))-f(s, x(s), \widetilde{\mathfrak{W}}(x)(s))| \mathrm{d} s \\
\leq & \frac{(\Psi(t)-\Psi(a))^{\mu}}{\Gamma(\mu+1)} \varepsilon+\frac{L_{f}}{\Gamma(\mu)} \int_{a}^{t} \mathbf{Q}_{\Psi}^{\mu}(t, s)\left\{|y(s)-x(s)|+L_{\widetilde{\mathfrak{W}}}|y(s)-x(s)|\right\} \mathrm{d} s \\
& \frac{(\Psi(t)-\Psi(a))^{\mu}}{\Gamma(\mu+1)} \varepsilon+\frac{L_{f}\left(1+L_{\widetilde{\mathfrak{W}}}\right)}{\Gamma(\mu)} \int_{a}^{t} \mathbf{Q}_{\Psi}^{\mu}(t, s)|y(s)-x(s)| \mathrm{d} s .
\end{aligned}
$$

Using the Gronwall lemma (Lemma 1) we get, for $\Delta$,

$$
|y(t)-x(t)| \leq \frac{(\Psi(t)-\Psi(a))^{\mu}}{\Gamma(\mu+1)} \varepsilon \mathcal{E}_{\mu}\left[\frac{L_{f}\left(1+L_{\widetilde{\mathfrak{W}}}\right)}{\Gamma(\mu)} \Gamma(\mu)(\Psi(t)-\Psi(a))^{\mu}\right] .
$$


$8^{1}$ J. VANTERLER DA C. SOUSA, ${ }^{2}$ KISHOR D. KUCCHE, AND ${ }^{3}$ E. CAPELAS DE OLIVEIRA

Therefore

$$
\begin{aligned}
\|y-x\|_{C_{1-\xi} ; \Psi} & =\sup _{t \in I}\left|(\Psi(t)-\Psi(a))^{1-\xi} y(t)-x(t)\right| \\
& \leq \frac{(\Psi(b)-\Psi(a))^{\mu+1-\xi}}{\Gamma(\mu+1)} \mathcal{E}_{\mu}\left[L_{f}\left(1+L_{\widetilde{\mathfrak{W}}}\right)(\Psi(b)-\Psi(a))^{\mu}\right] \varepsilon \\
& =c \varepsilon,
\end{aligned}
$$

where

$$
c:=\frac{(\Psi(b)-\Psi(a))^{\mu+1-\xi}}{\Gamma(\mu+1)} \mathcal{E}_{\mu}\left[L_{f}\left(1+L_{\widetilde{\mathfrak{W}}}\right)(\Psi(b)-\Psi(a))^{\mu}\right]
$$

with $\mathcal{E}_{\mu}(\cdot)$ a Mittag-Leffler function. This proves the solution of the problem Eq.(2.7) and Eq.(2.8) is Ulam-Hyers stable.

\section{Generalized Ulam-Hyers-Rassias stability}

In this section, another our main result investigate the uniqueness and stability of Ulam-Hyers-Rassias of the Cauchy fractional problem Eq.(2.7)-Eq.(2.8) on the infinite interval $I=[a, \infty)$.

Theorem 6. Consider the following:

$(\tilde{a}) f \in C_{1-\xi, \Psi}\left([a, \infty) \times \mathbb{R}^{2}, \mathbb{R}\right) ; \widetilde{\mathfrak{W}} \in C\left(\left(C_{1-\xi, \Psi} \Delta, \mathbb{R}\right),\left(C_{1-\xi, \Psi}(\Delta, \mathbb{R})\right)\right)$;

$(\tilde{b})$ There exists non-decreasing function $\widetilde{L}_{f} \in C\left([a, \infty), \mathbb{R}_{+}\right)$such that

$$
\left|f\left(t, u_{1}, u_{2}\right)-f\left(t, v_{1}, v_{2}\right)\right| \leq \widetilde{L}_{f}(t)\left(\left|u_{1}-v_{1}\right|+\left|u_{2}-v_{2}\right|\right)
$$

for $t \in[a, \infty)$, and $u_{i}, v_{i} \in \mathbb{R}$ with $i=1,2$;

$(\tilde{c})$ There exists non-decreasing function $\widetilde{L}_{\widetilde{\mathfrak{W}}} \in C\left([a, \infty), \mathbb{R}_{+}\right)$such that

$$
|\widetilde{\mathfrak{W}}(x)(t)-\widetilde{\mathfrak{W}}(y)(t)| \leq \widetilde{L}_{\widetilde{\mathfrak{W}}}(t)|x(t)-y(t)|
$$

for $x, y \in C_{1-\xi, \Psi}[a, \infty)$ and $t \in[a, \infty)$;

$(\tilde{d})$ The function $\varphi \in C_{1-\xi, \Psi}[a, \infty)$ is increasing;

(e) There exists $\lambda>0$ such that

$$
\frac{1}{\Gamma(\mu)} \int_{a}^{t} \mathbf{Q}_{\Psi}^{\mu}(t, s) \varphi(s) \mathrm{d} s \leq \lambda \varphi(t)
$$

with $t \in[a, \infty)$.

Then,

(1) the fractional problem Eq.(2.7)-Eq.(2.8) has a unique solution in $C_{1-\xi, \Psi}([a, \infty), \mathbb{R})$;

(2) the solution of the fractional problem Eq.(2.7)-Eq.(2.8) is generalized UlamHyers-Rassias stable with respect to $\varphi$.

Proof. With the conditions $(\tilde{a}),(\tilde{b})$ and $(\tilde{c})$ and following the steps in the proof of Theorem 5, one can easily prove that the problem Eq.(2.7)-Eq.(2.8), has a unique solution in $C_{1-\xi, \Psi}^{1}([a, \infty), \mathbb{R})$.

Let $y \in C_{1-\xi, \Psi}^{1}([a, \infty), \mathbb{R})$ satisfying the inequality (2.10). Let $x \in C_{1-\xi, \Psi}^{1}([a, \infty), \mathbb{R})$ is a unique solution of the following fractional Cauchy problem

$$
\left\{\begin{aligned}
{ }^{\mathbf{H}} \mathbf{D}_{a^{+}}^{\mu, \eta} x(t) & =f(t, x(t), \widetilde{\mathfrak{W}}(x)(t)), t \in[a, \infty) \\
\mathbf{I}_{a+}^{1-\xi, \Psi} x(a) & =y(a) .
\end{aligned}\right.
$$


ON THE ULAM-HYERS STABILITIES OF THE SOLUTIONS OF $\Psi$-HILFER FRACTIONAL DIFFERENTIAL EQUATION WITH

Then, its equivalent to the Volterra integral equation is

$$
x(t)=\mathbf{M}_{\xi}^{\Psi}(t, a) y(a)+\frac{1}{\Gamma(\mu)} \int_{a}^{t} \mathbf{Q}_{\Psi}^{\mu}(t, s) f(s, x(s), \widetilde{\mathfrak{W}}(x)(s)) \mathrm{d} s,
$$

with $t \in[a, \infty)$.

Since $y \in C_{1-\xi, \Psi}^{1}(\Delta, \mathbb{R})$ satisfies inequality (2.10), by means of the Remark 2.6. $y$ satisfies the following fractional integral inequality

$$
\begin{aligned}
& \quad\left|y(t)-\mathbf{M}_{\xi}^{\Psi}(t, a) y(a)-\frac{1}{\Gamma(\mu)} \int_{a}^{t} \mathbf{Q}_{\Psi}^{\mu}(t, s) f(s, x(s), \widetilde{\mathfrak{W}}(x)(s)) \mathrm{d} s\right| \\
& \leq \frac{1}{\Gamma(\mu)} \int_{a}^{t} \mathbf{Q}_{\Psi}^{\mu}(t, s) \varphi(s) \mathrm{d} s \leq \lambda \varphi(t)
\end{aligned}
$$

with $t \in[a, \infty)$.

From Eq.4.2) and Eq.4.3), we obtain

$$
\begin{aligned}
& |y(t)-x(t)| \\
= & \left|y(t)-\mathbf{M}_{\xi}^{\Psi}(t, a) y(a)-\frac{1}{\Gamma(\mu)} \int_{a}^{t} \mathbf{Q}_{\Psi}^{\mu}(t, s) f(s, x(s), \widetilde{\mathfrak{W}}(x)(s)) \mathrm{d} s\right| \\
\leq & \left|y(t)-\mathbf{M}_{\xi}^{\Psi}(t, a) y(a)-\frac{1}{\Gamma(\mu)} \int_{a}^{t} \mathbf{Q}_{\Psi}^{\mu}(t, s) f(s, y(s), \widetilde{\mathfrak{W}}(y)(s)) \mathrm{d} s\right|+ \\
& +\frac{1}{\Gamma(\mu)} \int_{a}^{t} \mathbf{Q}_{\Psi}^{\mu}(t, s)|f(s, y(s), \widetilde{\mathfrak{W}}(y)(s))-f(s, x(s), \widetilde{\mathfrak{W}}(x)(s))| \mathrm{d} s \\
\leq & \lambda \varphi(t)+\frac{1}{\Gamma(\mu)} \int_{a}^{t} \mathbf{Q}_{\Psi}^{\mu}(t, s) \widetilde{L}_{f}(s)\{|y(s)-x(s)|+\widetilde{\mathfrak{W}}(y)(s)-\widetilde{\mathfrak{W}}(x)(s)\} \mathrm{d} s \\
\leq & \lambda \varphi(t)+\frac{1}{\Gamma(\mu)} \int_{a}^{t} \mathbf{Q}_{\Psi}^{\mu}(t, s) \widetilde{L}_{f}(s)(1+\widetilde{L} \widetilde{\mathfrak{W}}(s))|y(s)-x(s)| \mathrm{d} s \\
\leq & \lambda \varphi(t)+\frac{\widetilde{L}_{f}(t)\left(1+\widetilde{L}_{\widetilde{\mathfrak{W}}}(t)\right)}{\Gamma(\mu)} \int_{a}^{t} \mathbf{Q}_{\Psi}^{\mu}(t, s)|y(s)-x(s)| \mathrm{d} s .
\end{aligned}
$$

Using the Gronwall lemma (Lemma 1), we have for $t \in[a, \infty)$

$$
|y(t)-x(t)| \leq \lambda \varphi(t) \mathcal{E}_{\mu}\left[\widetilde{L}_{f}(t)\left(1+\widetilde{L}_{\widetilde{\mathfrak{W}}}(t)\right)(\Psi(t)-\Psi(a))^{\mu}\right]
$$

Therefore

$$
(\Psi(t)-\Psi(a))^{1-\xi}|y(t)-x(t)| \leq c_{\varphi} \varphi(t), t \in[a, \infty)
$$

where $c_{\varphi}=\lambda \tilde{\Psi} \mathcal{E}_{\mu}(\tilde{K}), \tilde{\Psi}=\sup _{t \in[a, \infty)}\left|(\Psi(t)-\Psi(a))^{1-\xi}\right|<\infty$ and

$$
\tilde{K}=\sup _{t \in[a, \infty)}\left|\widetilde{L}_{f}(t)\left(1+\widetilde{L}_{\widetilde{\mathfrak{W}}}(t)\right)(\Psi(t)-\Psi(a))^{\mu}\right|<\infty .
$$

So, by Eq.(4.4), the solution of the problem Eq.(2.7)-Eq.(2.8) is generalized UlamHyers-Rassias stable with respect to $\varphi$.

We can conclude that the investigation that was carried out by means of Theorem 5 and Theorem [6] enabled a new class of Ulam-Hyers and Ulam-Hyers-Rassias stabilities on the compact interval $\Delta=[a, b]$ and on $I=[a, \infty)$. In this sense, the new results presented here are indeed important for the fractional calculus, in particular, for fractional analysis, enabling a growth in the area. In the following 
$10^{1}$ J. VANTERLER DA C. SOUSA, ${ }^{2}$ KISHOR D. KUCCHE, AND ${ }^{3}$ E. CAPELAS DE OLIVEIRA

section, we present a brief application of this new class of Ulam-Hyers stability, as particular cases of Theorem 5 and Theorem 6 .

\section{Application}

In this section as application, we will discuss a fractional Cauchy problem involving the Hadamard derivative and the stability of its solution, in both cases, Ulam-Hyers and generalized Ulam-Hyers-Rassias. The results are presented as theorems.

Consider the following fractional Cauchy problem

$$
{ }^{H D} \mathcal{D}_{a_{+}}^{\mu} x(t)=f\left(t, x(t), \frac{1}{\Gamma(\mu)} \int_{a}^{t} \ln \left(\frac{t}{s}\right)^{\mu-1} K(t, s, x(s)) \mathrm{d} s\right), \quad t \in I
$$

where $I=[0,1]$ or $[0, \infty),{ }^{H D} \mathcal{D}_{a_{+}}^{\mu}(\cdot)$ is the Hadamard fractional derivative and the inequalities

for $t \in I$.

$$
\begin{gathered}
\left|{ }^{H D} \mathcal{D}_{a_{+}}^{\mu} y(t)-f\left(t, y(t), \frac{1}{\Gamma(\mu)} \int_{a}^{t} \ln \left(\frac{t}{s}\right)^{\mu-1} K(t, s, y(s)) \mathrm{d} s\right)\right| \leq \varepsilon, \\
\left|{ }^{H D} \mathcal{D}_{a_{+}}^{\mu} y(t)-f\left(t, y(t), \frac{1}{\Gamma(\mu)} \int_{a}^{t} \ln \left(\frac{t}{s}\right)^{\mu-1} K(t, s, y(s)) \mathrm{d} s\right)\right| \leq \varphi(t)
\end{gathered}
$$

In this case, Theorem 5 and Theorem 6 become

Theorem 7. Suppose that

(A1) $f \in C_{1-\xi, \Psi}\left([0,1] \times \mathbb{R}^{2}, \mathbb{R}\right) ; \widetilde{\mathfrak{W}} \in C_{1-\xi, \Psi}\left(\left(C_{1-\xi, \Psi}[a, b], \mathbb{R}\right),\left(C_{1-\xi, \Psi}[a, b], \mathbb{R}\right)\right)$;

(A2) There exists $L_{f}>0$ such that, for all $t \in[0,1]$,

$$
\left|f\left(t, u_{1}, u_{2}\right)-f\left(t, v_{1}, v_{2}\right)\right| \leq L_{f} \sum_{i=1}^{2}\left|u_{i}-v_{i}\right|
$$

with $u_{i}, v_{i} \in \mathbb{R}$ and $i=1,2$;

0 (A3) There exists $L_{K}>0$ such that

$$
|K(t, s, x(s))-K(t, s, y(s))| \leq L_{K}|x(t)-y(t)|
$$

with $x, y \in C_{1-\xi, \Psi}[0,1]$ and $t \in[0,1]$.

$$
\text { (A4) } \frac{\left(\ln \left(\frac{b}{a}\right)\right)^{\mu}}{\Gamma(2 \mu)} \Gamma(\mu) L_{f}\left(1+L_{K}\right)<1 \text {. }
$$

Then

(i) The problem Eq.(2.7)-Eq.(2.8) has a unique solution in $C_{1-\xi, \Psi}([0,1], \mathbb{R})$.

(ii) The solution of Eq.(2.7) is Ulam-Hyers stable.

Proof. The proof follows the same steps as in Theorem 5 .

Theorem 8. We assume that

(B1) $f \in C_{1-\xi, \Psi}\left([0, \infty) \times \mathbb{R}^{2}, \mathbb{R}\right) ; \widetilde{\mathfrak{W}} \in C\left(\left(C_{1-\xi, \Psi}[0,1], \mathbb{R}\right),\left(C_{1-\xi, \Psi}[0,1], \mathbb{R}\right)\right)$;

(B2) There exists non-decreasing function $\widetilde{L}_{f} \in C\left([0, \infty), \mathbb{R}_{+}\right)$such that, for all $t \in[0, \infty]$,

$$
\left|f\left(t, u_{1}, u_{2}\right)-f\left(t, v_{1}, v_{2}\right)\right| \leq \widetilde{L}_{f}(t)\left(\left|u_{1}-v_{1}\right|+\left|u_{2}-v_{2}\right|\right)
$$

with $u_{i}, v_{i} \in \mathbb{R}$ and $i=1,2$; 
(B3) There exists non-decreasing function $\widetilde{L}_{K} \in C([0, \infty), \mathbb{R})$ such that

$$
\mid K(t, s, u))-K(t, s, v)\left|\leq \widetilde{L}_{K}(t)\right| x(t)-y(t) \mid
$$

with $x, y \in C_{1-\xi, \Psi}[0, \infty)$ and $t \in[0, \infty)$.

(B4) The function $\varphi \in C_{1-\xi, \Psi}[0, \infty)$ is increasing;

(B5) There exists $\lambda>0$ such that

$$
\frac{1}{\Gamma(\mu)} \int_{a}^{t} \ln \left(\frac{t}{s}\right)^{\mu-1} \varphi(s) \mathrm{d} s \leq \lambda \varphi(t), \quad t \in[0, \infty)
$$

Then

(i) The problem Eq.(2.7)-Eq.(2.8) has a unique solution in $C_{1-\xi, \Psi}([0, \infty), \mathbb{R})$.

(ii) The solution of Eq.(2.7) is Ulam-Hyers-Rassias stable with respect to $\varphi$.

Proof. The proof follows the same steps as in Theorem 6 .

\section{Concluding Remarks}

The investigation of Ulam-Hyers stabilities of solutions of several types of fractional differential equations is a major motivation for researchers. Here we investigated a new class of Ulam-Hyers stabilities of the differential equation with abstract Volterra operator introduced by means of the $\Psi$-Hilfer fractional derivative on the compact interval $\Delta=[a, b]$ and on the infinite interval $I=[a, \infty)$, making use of Banach's fixed point theorem and Gronwall inequality. In this paper, the results were obtained in the Banach space. It will be whether there is a possibility of investigating such results in other spaces, such as Fréchet and fractional Sobolev spaces. Researches in this follow-up are being worked on and future works will be published.

\section{ACKNOWLEDGMENT}

\section{I support financial support of the PNDP-CAPES scholarship of the Pos-Graduate Program in Mathematics UFPA/UFAM.}

\section{REFERENCES}

1. S. Kantorovitz, K.-J. Pei, Pairs of operators satisfying the Volterra commutation relation, Indiana University Math. J. 23 (12) (1974) 1177-1197.

2. C. Corduneanu, Abstract Volterra equations: a survey, Math. and Comput. Model. 32 (11-13) (2000) 1503-1528.

3. C. Corduneanu, Neutral functional differential equations with abstract Volterra operators, Adv. in Nonlinear Dyn. 2 (4) (1997) 229.

4. C. Corduneanu, Existence of solutions for neutral functional differential equations with causal operators, J. Diff. Equa. 168 (1) (2000) 93-101.

5. M. Vath, Linear and nonlinear abstract Volterra equations, Funct. Diff. Equa. 5 (3-4) (2004) $\mathrm{p}-451$.

6. D. M. Bedivan, D. O' Regan, Fixed point sets for abstract Volterra operators on Fréchet spaces, Applicable Analysis 76 (1-2) (2000) 131-152.

7. M. A. Serban, I. A. Rus, A. Petrusel, A class of abstract Volterra equations, via weakly Picard operators technique, Math. Inequal. Appl 13 (2) (2010) 255-269.

8. D. Guo, Impulsive integral equations in Banach spaces and applications, Inter. J. Stochastic Anal. 5 (2) (1992) 111-122.

9. D. Guo, V. Lakshmikantham, X. Liu, Nonlinear integral equations in abstract spaces, Vol. 373, Kluwer Academic Publishers, Dordrecht, The Netherlands, 1996.

10. V. Kolmanovskii, A. Myshkis, Applied theory of functional differential equations, Vol. 85, Kluwer Academic Publishers, Dordrecht, The Netherlands, 1992. 
11. D. H. Hyers, G. Isac, T. Rassias, Stability of functional equations in several variables, Birkhäuser, Boston, 1998.

12. S.-M. Jung, A fixed point approach to the stability of a Volterra integral equation, Fixed Point Theory and Appl. 2007 (1) (2007) 057064.

13. T. M. Rassias, On the stability of the linear mapping in Banach spaces, Proceedings of the American Mathematical Society 72 (2) (1978) 297-300.

14. I. A. Rus, Ulam stability of ordinary differential equations, Stud. Univ. "Babeş-Bolyai" Math. (54) (2009) 125-133.

15. D. Otrocol, Ulam stabilities of differential equation with abstract Volterra operator in a Banach space, Nonlinear Funct. Anal. Appl. 15 (4) (2010) 613-619.

16. W. Wei, X. Li, X. Li, New stability results for fractional integral equation, Comput. \& Math. Appl. 64 (10) (2012) 3468-3476.

17. M. Kostic, Abstract Volterra integro-differential equations, Taylor and Francis Group/CRC Press, 2015.

18. K. Oldham, J. Spanier, The Fractional Calculus Theory and Applications of Differentiation and Integration to Arbitrary Order, Vol. 111, Elsevier, Academic Press, New York, 1974.

19. Z. Yong, W. Jinrong, Z. Lu, Basic theory of fractional differential equations, World Scientific Publishing Company, Singapore, New Jersey, London and Hong Kong, 2014.

20. R. Herrmann, Fractional Calculus: An Introduction for Physicists, World Scientific Publishing Company, Singapore, 2011.

21. I. Podlubny, Fractional Differential Equations, Mathematics in Science and Engineering, Academic Press, San Diego, Vol. 198, 1999.

22. S. G. Samko, A. A. Kilbas, O. I. Marichev, Fractional Integrals and Derivatives, Theory and Applications, Gordon and Breach, New York, 1993.

23. A. A. Kilbas, H. M. Srivastava, J. J. Trujillo, Theory and Applications of Fractional Differential Equations, Vol. 204, Elsevier, Amsterdam, 2006.

24. J. Vanterler da C. Sousa, E. Capelas de Oliveira, On the $\psi$-Hilfer fractional derivative, Commun. Nonlinear Sci. Numer. Simulat. 60 (2018) 72-91.

25. J. Vanterler da C. Sousa, E. Capelas de Oliveira, On a new operator in fractional calculus and applications, arXiv:1710.03712, (2018).

26. J. A. David, D. D. Quintino, C. M. C. Inacio Jr, J. A. T. Machado, Fractional dynamic behavior in ethanol prices series, J. Comput. and Appl. Math. 339 (2018) 85-93.

27. A. Dabiri, B. P. Moghaddam, J. A. T. Machado, Optimal variable-order fractional PID controllers for dynamical systems, J. Comput. and Appl. Math. 339 (2018) 40-48.

28. S. Jahanshahi, E. Babolian, D. F. M. Torres, A. R. Vahidi, A fractional Gauss-Jacobi quadrature rule for approximating fractional integrals and derivatives, Chaos, Solitons \& Fractals 102 (2017) 295-304.

29. D. Tavares, R. Almeida, D. F. M. Torres, Combined fractional variational problems of variable order and some computational aspects, J. Comput. and Appl. Math. 339 (2018) 374-388.

30. A. B. Salati, M. Shamsi, D. F. M. Torres, Direct transcription methods based on fractional integral approximation formulas for solving nonlinear fractional optimal control problems, Commun. Nonlinear Sci. Numer. Simulat. 67 (2019) 334-350.

31. J. Vanterler da C. Sousa, M. N. N. dos Santos, L. A. Magna, E. Capelas de Oliveira, Validation of a fractional model for erythrocyte sedimentation rate, Comp. Appl. Math. (2018). https://doi.org/10.1007/s40314-018-0717-0.

32. S. Abbas, M. Benchohra, J. E. Lagreg, A. Alsaedi, Y. Zhou, Existence and Ulam stability for fractional differential equations of Hilfer-Hadamard type, Adv. Diff. Equa. 2017 (1) (2017) 180 .

33. S. Abbas, M. Benchohra, J. R. Graef, J. Henderson, Implicit Fractional Differential and Integral Equations: Existence and Stability, Vol. 26, Walter de Gruyter GmbH \& Co KG, Berlin, Germany, 2018.

34. J. Wang, M. Fečkan, Y. Zhou, A survey on impulsive fractional differential equations, Fract. Cal. Appl. Anal. 19 (4) (2016) 806-831.

35. J. Wang, M. Fec, Y. Zhou, Ulam's type stability of impulsive ordinary differential equations, J. Math. Anal. Appl. 395 (1) (2012) 258-264.

36. J. Vanterler da C. Sousa, E. Capelas de Oliveira, Ulam-Hyers stability of a nonlinear fractional Volterra integro-differential equation, Appl. Math. Lett. 81 (2018) 50-56. 
ON THE ULAM-HYERS STABILITIES OF THE SOLUTIONS OF $\Psi$-HILFER FRACTIONAL DIFFERENTIAL EQUATION WITH

37. J. Vanterler da C. Sousa, E. Capelas de Oliveira, On the Ulam-Hyers-Rassias stability for nonlinear fractional differential equations using the $\psi$-Hilfer operator, J. Fixed Point Theory and Appl. 20 (3) (2018) 96.

38. E. Capelas de Oliveira, J. Vanterler da C. Sousa, Ulam-Hyers-Rassias stability for a class of fractional integro-differential equations, Results Math. 73 (3) (2018) 111.

39. J. Vanterler da C. Sousa, K. D. Kucche, E. Capelas de Oliveira, Stability of $\psi$-Hilfer impulsive fractional differential equations, Appl. Math. Lett. 88 (2019) 73-80.

40. J. Vanterler da C. Sousa, E. Capelas de Oliveira, Fractional order pseudoparabolic partial differential equation: Ulam-Hyers stability, Bull. Braz. Math. Soc., New Series (2018) 1-16.

41. J. Vanterler da C. Sousa, E. Capelas de Oliveira, F. G. Rodigues, Stability of the fractional Volterra integro-differential equation by means of $\psi$-Hilfer operator, arXiv:1804.02601.

42. J. Vanterler da C. Sousa, D. S. Oliveira, E. Capelas de Oliveira, On the existence and stability for impulsive fractional integrodifferential equation, arXiv:1806.01442.

43. J. Vanterler da C. Sousa, E. Capelas de Oliveira, A Gronwall inequality and the Cauchy-type problem by means of $\psi$-Hilfer operator, arXiv:1709.03634.

1 Mathematics faculty, Federal University of Pará,, Augusto Corrêa Street, NumBer 01, 66075-110, BelÉm, PA, Brazil

E-mail address: ra160908@ime.unicamp.br

${ }^{2}$ Department of Mathematics, Shivaji University,, Kolhapur 416004 , Maharashtra, INDIA

E-mail address: kdkucche@gmail.com

3 Department of Applied Mathematics, Imecc-Unicamp,, 13083-859, Campinas, SP, BRAZIL

E-mail address: capelas@ime.unicamp.br 